\title{
Algunas tendencias de la formación bibliotecológica en América Latina
}

\author{
María Teresa Múnera Torres
}

Artículo recibido:

17 de mayo de 2015

Artículo aceptado:

2 de mayo de 2016

\section{Resumen}

Con base en la investigación "La terminología de la bibliotecología en América Latina: un estudio comparativo", se presenta un panorama sobre algunos de los aspectos del estado de la formación bibliotecológica en universidades de países como Argentina, Brasil, Chile, Colombia, Costa Rica, Guatemala, México, Paraguay, Perú, Uruguay y Venezuela. Se abordan aspectos relacionados con la evolución de la formación bibliotecológica, las áreas del conocimiento de las cuales depende la educación bibliotecológica en América Latina, así como los nombres que recibe este tipo de formación en las universidades que hacen parte de la muestra de la investigación.

\footnotetext{
Universidad de Antioquia, Colombia._maria.munera@udea.edu.com mmuner1@yahoo.es

INVESTIGACIÓN BIBLIOTECOLÓGICA, vol.32, núm.74, enero/marzo, 2018, México,
} ISSN: 2448-8321. pp. 101-122 
Se aclara además que para efectos del presente documento, se otorgará tanto a la bibliotecología como a la biblioteconomía la condición de sinónimos, razón por la cual aparecerán textos en los que se contemplen ambos términos indistintamente.

Palabras clave: Formación Bibliotecológica - América Latina; Educación Bibliotecológica - América Latina; Bibliotecología - Terminología

Some trends in library and information science training in Latin America

Maria-Teresa Múnera-Torres

\begin{abstract}
Touching on the research paper: "Terminology of library and information science in Latin America: a comparative study"; this article presents an overview of some aspects of the state of library and information science training at universities in Argentina, Brazil, Chile, Colombia, Costa Rica, Guatemala, Mexico, Paraguay, Peru, Uruguay and Venezuela. The paper examines aspects of the development of library and information science training, the fields of knowledge governing librarianship education in Latin America, as well as the names given to this type of training in universities included in the research sample. For purposes of this research report, library science and librarianship are considered synonymous. As such, research employing either term is included in the research sample.
\end{abstract}

Keywords: Library And Information Science Training - Latin America; Library And Information Science Education - Latin America; Library and Information Science - Terminology 


\section{INTRODUCCIÓN}

$\mathrm{E}^{\mathrm{n}}$ el presente documento se pretende dar cuenta sobre algunos de los hallazgos a los que se llega con el desarrollo del estudio comparativo de la terminología de la bibliotecología en América Latina (Múnera Torres, 2014: 111) y que se lleva a cabo en 11 países, 40 universidades y 47 programas de educación bibliotecológica. ${ }^{1}$ De forma específica se enfoca en componentes de la formación bibliotecológica en los que se identifican las áreas del conocimiento de las que depende dicha formación en América Latina, así como los nombres que recibe la misma en los países que conforman la muestra de la investigación, no sin antes hacer una introducción sobre los orígenes y antecedentes de la evolución de los procesos de educación bibliotecológica dentro de un contexto general. Por tanto, se abordan tópicos que forman parte de la educación bibliotecológica, ya que en los últimos años se han hecho estudios en los que se plantea interés por parte de académicos como José López Yepes, María Luisa Orera Orera, Miguel Ángel Rendón Rojas, Jaime Ríos Ortega, Agustín Gutiérrez Chiñas, entre otros, sobre la variación denominativa que se manifiesta en el ámbito de la bibliotecología.

Con el fin de lograr una idea precisa sobre la muestra que se define para la investigación, la Tabla 1 indica los países, las universidades y los programas que la conforman. Asimismo, se incluye una tabla al final del artículo en la que se registran los países, universidades y programas de formación bibliotecológica específicos.

\begin{tabular}{|l|c|c|}
\hline País & Universidades & $\begin{array}{c}\text { Programas } \\
\text { de formación } \\
\text { bibliotecológica }\end{array}$ \\
\hline 1. Argentina & 3 & 5 \\
\hline 2. Brasil & 21 & 25 \\
\hline 3. Chile & 1 & 1 \\
\hline 4. Colombia & 3 & 3 \\
\hline 5. Costa Rica & 1 & 2 \\
\hline 6. Guatemala & 1 & 1 \\
\hline 7. México & 4 & 4 \\
\hline 8. Paraguay & 1 & 1 \\
\hline
\end{tabular}

1 En cuanto al nombre de las universidades y programas de formación bibliotecológica que conforman la muestra, la relación correspondiente se presenta en la tabla anexa al final del artículo. 


\begin{tabular}{|l|c|c|}
\hline 9. Perú & 2 & 2 \\
\hline 10. Uruguay & 1 & 1 \\
\hline 11. Venezuela & 2 & 2 \\
\hline Totales & 40 & 47 \\
\hline
\end{tabular}

Tabla 1. Relación de países y número de universidades y programas muestra del estudio comparativo de la terminología de la bibliotecología en América Latina

Fuente: Múnera Torres, 2016:133

Como se aprecia, Brasil presenta un mayor número de universidades y programas de formación bibliotecológica, lo que marca una pauta para los estudios comparativos. Por supuesto, las propuestas de formación bibliotecológica de Brasil se constituyen en referentes que muy seguramente señalan una tendencia dentro de las nuevas dinámicas de este tipo de formación para América Latina.

\section{UNA MIRADA A LOS INICIOS DE LA FORMACIÓN BIBLIOTECOLÓGICA}

En los albores de la bibliotecología se aprecia el predominio de la labor empírica por parte de quienes están al frente de las bibliotecas. Aún no se aprecian propuestas de formación en el ámbito bibliotecológico, sino una actividad práctica desempeñada por intelectuales, eruditos y excelentes lectores. Las bibliotecas se constituyen en espacios dedicados más a la función de depósitos que a la de ser lugares de proyección y servicio a posibles usuarios de la información, lo que se refleja en que la bibliotecología en sus inicios se centra en la labor de conservación de los soportes del conocimiento de las bibliotecas y quienes se desempeñan en ellas. Es así como la investigadora Luisa Orera Orera se refiere a esta época como a la etapa precientífica del desarrollo de la bibliotecología:

Algunos autores han distinguido una etapa que denominan pre científica, que se corresponde con bibliotecas consistentes en depósitos de tesoros bibliográficos y unos bibliotecarios erudito-conservadores. A esta fase corresponde una dilatada etapa histórica de las bibliotecas que nacen en el mundo oriental y se extenderán al occidente. (1995: 74)

La función de erudición y de cuidado de los fondos de las bibliotecas se transforma con el paso del tiempo y entran en escena dinámicas que plantean la necesidad de iniciar propuestas de educación formal para quienes se desempeñen 
en estos recintos. Durante el siglo XIX, época en la que se manifiesta la llamada biblioteconomía científica, inician una serie de escuelas en las que se imparte la formación en el ámbito bibliotecológico, tal como lo señala la misma Luisa Orera Orera: "El comienzo de la enseñanza formal de la Biblioteconomía, coincide, como es lógico con el nacimiento de la Biblioteconomía científica en el siglo XIX. Las primeras escuelas, que de una u otra forma se ocupan de la formación de bibliotecarios nacen en esta centuria” (2002: 44).

Es así como se empiezan a gestar escuelas de formación bibliotecológica en países de Europa y América. Se hace alusión a las mismas en la presentación de las ofertas de educación bibliotecológica de la Universidad de Buenos Aires, en Argentina: "Hubo dos hechos que sucedieron a principios del siglo XIX: la creación en 1822 de L'Ecole des Chatres en Paris y en 1883 la Escuela de Columbia en Estados Unidos, las cuales representaban dos corrientes distintas en su formación, la primera humanística y la segunda profundamente técnica" (Universidad de Buenos Aires, 2010). Y es que durante el proceso de desarrollo de la educación bibliotecológica se generan diversos enfoques dentro de la misma. Es por ello que algunas escuelas se enfocan en lo técnico y otras en lo social y humanístico, debido a que consideran el desarrollo de la actividad bibliotecológica como un campo del saber que se relaciona directamente con procesos que contribuyen con el crecimiento de los individuos y de la sociedad.

Uno de los mayores exponentes de la tendencia social es precisamente Jesse H. Shera, a quien José López Yepes se refiere en su Diccionario Enciclopédico de Ciencias de la Documentación en los siguientes términos: "Quizá el más brillante de los autores de la Escuela de Chicago, Jesse H. Shera, considera la Biblioteconomía como una ciencia social, a la vez que la más interdisciplinar de todas las disciplinas" (2004: 227).

Durante el siglo XX se manifiestan una serie de fenómenos que inciden en el desarrollo de la bibliotecología y, por supuesto, en los procesos de formación. Entre los más destacados están el origen y desarrollo de la documentación como campo relacionado con la bibliotecología, así como el de la informática, que transforma de manera radical muchas de las dinámicas del hacer bibliotecológico y que con la introducción de las tecnologías de la información y la comunicación se constituyen en componentes fundamentales de lo que se conoce como la sociedad del conocimiento y de la información.

Los cambios que se introducen durante este periodo contribuyen al fortalecimiento de los procesos de formación bibliotecológica desde la investigación y a la generación de nuevo conocimiento en torno a la bibliotecología, así como con áreas relacionadas, de manera especial en los países de América Latina. Al respecto Lina Escalona Ríos expresa: 
La educación bibliotecológica en América Latina, es una profesión relativamente joven. En toda la región es a mediados del siglo XX que se establecen los primeros programas académicos en diversas universidades, especialmente públicas. Con un desarrollo incipiente, las instituciones educativas se han visto envueltas en cambios sociales y económicos radicales que han afectado la profesión bibliotecológica; desde la corriente de erudición bajo la cual surgen los primeros programas hasta el desarrollo de las TIC y el proceso globalizador que se da a partir de la década de los 70, lo que ha hecho que se den cambios curriculares radicales y constantes. (2011:203)

Como se observa, la formación bibliotecológica se empieza a consolidar durante el siglo XX y en este periodo se definen una serie de enfoques que orientan la educación bibliotecológica con base en influencias de tipo educativo, social, económico, político, científico y tecnológico. Esto influye en el desarrollo teórico e investigativo de la bibliotecología y la ubica como una ciencia que presenta un objeto de estudio propio, con líneas teóricas de investigación y en torno a la cual se gestan diversas propuestas de orientación y de énfasis. Al respecto Emilio Setién Quesada expresa los siguientes planteamientos:

El futuro de la teoría bibliotecológica marchará, como ciencia social, por diversas vías en virtud de las variadas tendencias aparecidas durante el siglo XX en el tratamiento teórico de la profesión, y de las que puedan surgir en el siglo XXI, así como de las exigencias que se le presenten a los distintos niveles del trabajo creador del bibliotecario. Influirán también en esa diversidad, de forma determinante, las situaciones de carácter económico, político, histórico y cultural que predominen en el entorno. O sea, que esos derroteros resultarán de las múltiples combinaciones que surjan entre los componentes de las tendencias teóricas de la bibliotecología, los niveles de creación del trabajo bibliotecario y las condiciones económico sociales concretas que prevalezcan en el entorno del fenómeno bibliotecario. (2002: 41)

Es indiscutible el grado de evolución que evidencia la dinámica de la educación bibliotecológica y los componentes que se logran aglutinar en torno a la ciencia bibliotecológica actual. Por otra parte, resulta interesante identificar las áreas del conocimiento de las que depende la formación bibliotecológica, a fin de contar con una idea específica sobre las tendencias que se manifiestan sobre el particular desde esta perspectiva. 


\section{ÁreAs DEL CONOCIMIENTO DE LAS QUE DEPENDE LA FORMACIÓN Bibliotecológica en AmÉrica LATina}

Como parte del estudio investigativo, se visualizan las áreas del conocimiento en las cuales la formación bibliotecológica se encuentra incluida como campo temático específico. Entre las áreas más notables se identifican el arte, la comunicación social, la gestión de la información, la ciencia de la información, las ciencias de la documentación, las ciencias económicas y sociales, las ciencias de la educación, las ciencias humanas, las ciencias sociales, las ciencias de la información, la filosofía y letras, la filosofía y humanidades, la información, entre otras. No obstante, es interesante resaltar el hecho de que la misma bibliotecología se constituye también en una de las áreas de las cuales depende la formación bibliotecológica actual.

También es pertinente aclarar que las áreas del conocimiento de las cuales depende la formación bibliotecológica se toman tanto de las facultades como de los centros, departamentos, institutos y demás estructuras administrativas de las que depende este tipo de formación, razón por la cual se obtiene un mayor número de programas de formación bibliotecológica que dependen de las distintas áreas del saber, lo que por supuesto supera el número de programas definidos dentro de la muestra de la investigación.

Se recuerda, igualmente, que al ser una muestra de programas brasileños muchos de los datos arrojados se basan en la información proporcionada por las universidades brasileñas que conforman la muestra. Por tanto, y luego de realizar un análisis comparativo sobre el área del conocimiento de la cual existe una mayor dependencia de la formación bibliotecológica, se obtiene el siguiente resultado: las áreas del conocimiento de las cuales depende en mayor medida la formación bibliotecológica son la ciencia de la información, la bibliotecología, la biblioteconomía, la filosofía y letras y las humanidades. Este resultado se aprecia más claramente en la Tabla 2.

\begin{tabular}{|l|c|}
\hline \multicolumn{2}{|c|}{ Áreas del conocimiento de las que depende la formación bibliotecológica en América Latina } \\
\hline Áreas del Conocimiento & $\begin{array}{c}\text { Número de programas de } \\
\text { fornación bibliotecológica }\end{array}$ \\
\hline Arte y Comunicación Social & 1 \\
\hline Artes y Comunicación & 2 \\
\hline Bibliotecología & 8 \\
\hline Bibliotecología y Archivología & 2 \\
\hline Bibliotecología y Ciencia de la Información & 2 \\
\hline Bibliotecología y Ciencias Afines & 1 \\
\hline
\end{tabular}




\begin{tabular}{|c|c|}
\hline Bibliotecología y Ciencias de la Información & 1 \\
\hline Bibliotecología, Documentación e Información & 2 \\
\hline Biblioteconomía & 6 \\
\hline Bibliotecología y Comunicación & 1 \\
\hline Bibliotecología y Documentación & 1 \\
\hline Ciencia y Gestón de la Información & 1 \\
\hline Ciencia de la Información & 10 \\
\hline Ciencias de la Documentación & 1 \\
\hline Ciencias Económicas y Sociales & 1 \\
\hline Ciencias de la Educación & 1 \\
\hline Ciencias Humanas, Comunicación y Arte & 1 \\
\hline Ciencias Humanas y de Educación & 3 \\
\hline Ciencias Humanas y de la Información & 1 \\
\hline Ciencias Humanas y Sociales & 1 \\
\hline Ciencias Humanas y Sociales Aplicadas & 1 \\
\hline Ciencias de la Información & 2 \\
\hline Ciencias Sociales & 1 \\
\hline Ciencias Sociales Aplicadas & 3 \\
\hline Comunicación y Biblioteconomía & 2 \\
\hline Comunicación y Lenguaje & 1 \\
\hline Comunicación y Artes & 1 \\
\hline Educación y Ciencias Humanas & 1 \\
\hline Educación, Comunicación y Artes & 1 \\
\hline Educación, Información y Comunicación & 1 \\
\hline Filosofía y Ciencias & 1 \\
\hline Filosofía, Ciencias y Letras & 1 \\
\hline Filosofía y Humanidades & 1 \\
\hline Filosofía y Letras & 6 \\
\hline Humanidades & 4 \\
\hline Humanidades y Ciencias de la Educación & 2 \\
\hline Humanidades y Educación & 2 \\
\hline Información & 1 \\
\hline Información y Comunicación & 1 \\
\hline Letras y Ciencias Humanas & 2 \\
\hline Politécnica & 1 \\
\hline
\end{tabular}

Tabla 2. Áreas del conocimiento de las que depende la formación bibliotecológica Fuente: Múnera Torres, 2016: 145 
$\mathrm{Al}$ identificar las áreas del conocimiento de las cuales depende la formación bibliotecológica en América Latina, de forma específica en las universidades que conforman la muestra de la investigación, se aprecia cómo aun cuando son numerosas las áreas del saber de las cuales depende dicha formación se identifica a la misma bibliotecología, así como a la biblioteconomía, la documentación, la ciencia de la información, las ciencias de la información, entre otras, como líderes en este aspecto que ha sido tomado como variable dentro de la investigación.

También resulta interesante observar cómo las áreas del conocimiento de las cuales depende la formación bibliotecológica que arrojan un mayor porcentaje son la ciencia de la información, la bibliotecología y la biblioteconomía, seguidas de la filosofía y letras y de las humanidades. Con este resultado, se visualiza la tendencia de existir un mayor grado de dependencia a nivel de conocimiento de áreas relacionadas con la bibliotecología, y que la dependencia de áreas del saber diferentes se ve reducida en contraste con la de las áreas bibliotecológicas y de la información.

En consecuencia, se puede considerar que las áreas del conocimiento que rigen la formación bibliotecológica pertenecen a la misma bibliotecología, biblioteconomía y ciencia de la información. Con ello se observa una interesante realidad sobre el liderazgo de conocimiento que puede ejercer la ciencia bibliotecológica y de la información como componente fundamental del desarrollo de la bibliotecología en América Latina, no obstante depender jerárquicamente de unidades académicas que corresponden a campos del conocimiento diferentes.

Se constata igualmente cómo los programas de formación bibliotecológica de Brasil marcan la línea en este sentido, ya que en dicho país la educación bibliotecológica, al denominarse como biblioteconomía, se enfoca hacia el estudio de la ciencia de la información, y en muchos casos se complementa con la misma biblioteconomía, razón por la cual el resultado del análisis de esta variable arroja a la ciencia de la información como área que lidera la jerarquía de conocimiento del cual depende la formación bibliotecológica en América Latina.

Este resultado obedece especialmente al desarrollo e introducción de nuevas dinámicas dentro de los procesos de formación bibliotecológica en América Latina, así como a los nuevos paradigmas de desempeño profesional que se manifiestan en este ámbito específico del saber, con base en el advenimiento de las tecnologías de la información y la comunicación como componentes de las nuevas formas de desempeño de los profesionales de la bibliotecología, entre las cuales se identifican, además de las tradicionales, las de docencia, investigación, emprendimiento y otras que han hecho trascender y crecer aún 
más este campo del conocimiento como componente fundamental para el desarrollo de la sociedad.

No obstante haber identificado las áreas del conocimiento de las cuales depende la formación bibliotecológica dentro de la muestra elegida para la investigación, resulta interesante observar en cada uno de los países objeto de estudio cuáles son las áreas del conocimiento específicas de las que depende la formación bibliotecológica que se imparte en la actualidad, de acuerdo con la información que se ilustra en la Tabla 3.

\begin{tabular}{|c|c|c|}
\hline País & $\begin{array}{l}\text { Áreas del conocimiento que rigen la formacón } \\
\text { bibliotecológica en América Latina }\end{array}$ & $\begin{array}{l}\text { Área del conocimiento de las } \\
\text { que más depende la forma- } \\
\text { ción bibliotecológica }\end{array}$ \\
\hline Argentina & $\begin{array}{l}\text { Bibliotecología (2) } \\
\text { Bibliotecología y Ciencias de la Información (2) } \\
\text { Filosofía y Humanidades (1) } \\
\text { Filosofía y Letras (1) } \\
\text { Humanidades y Ciencias de la Educación (1) }\end{array}$ & Bibliotecología \\
\hline Brasil & $\begin{array}{l}\text { Artes y Comunicación (1) } \\
\text { Artes y Comunicación Social (1) } \\
\text { Biblioteconomía (5) } \\
\text { Biblioteconomía y Comunicación (1) } \\
\text { Biblioteconomía y Documentación (2) } \\
\text { Ciencia de la Información (8) } \\
\text { Ciencias de la Información (1) } \\
\text { Ciencia y Gestión de la Información (1) } \\
\text { Ciencias de la Educación (1) } \\
\text { Ciencias Humanas, Comunicación y Arte (1) } \\
\text { Ciencias Humanas y de Educación (1) } \\
\text { Ciencias Humanas y de la Información (1) } \\
\text { Ciencias Humanas y Sociales (3) } \\
\text { Ciencias Sociales y Aplicadas (3) } \\
\text { Comunicación y Artes (1) } \\
\text { Comunicación y Biblioteconomía (1) } \\
\text { Educación y Ciencias Humanas (1) } \\
\text { Educación, Comunicación y Artes (1) } \\
\text { Educación, Información y Comunicación (1) } \\
\text { Filosofía y Ciencias (1) } \\
\text { Filosofía, Ciencias y Letras (1) } \\
\text { Humanidades (1) } \\
\text { Información y Documentación (1) }\end{array}$ & $\begin{array}{l}\text { Ciencias de la Información } \\
\text { Biblioteconomía }\end{array}$ \\
\hline Chile & $\begin{array}{l}\text { Ciencias Sociales } \\
\text { Ciencias de la Documentación }\end{array}$ & $\begin{array}{l}\text { Ciencias Sociales } \\
\text { Ciencias de la Documentación }\end{array}$ \\
\hline Colombia & $\begin{array}{l}\text { Bibliotecología } \\
\text { Ciencias de la Información } \\
\text { Ciencias Económicas Sociales } \\
\text { Comunicación y Lenguaje }\end{array}$ & $\begin{array}{l}\text { Bibliotecología } \\
\text { Comunicación y Lenguaje } \\
\text { Ciencia de la Información } \\
\text { Ciencias Económicas Sociales }\end{array}$ \\
\hline
\end{tabular}




\begin{tabular}{|l|l|l|}
\hline Costa Rica & $\begin{array}{l}\text { Filosofía y Letras (2) } \\
\text { Bibliotecología, Documentación e Información (2) }\end{array}$ & $\begin{array}{l}\text { Filosofía y Letras } \\
\text { Bibliotecología, Documentación e } \\
\text { Información }\end{array}$ \\
\hline Guatemala & $\begin{array}{l}\text { Humanidades } \\
\text { Bibliotecología }\end{array}$ & $\begin{array}{l}\text { Humanidades } \\
\text { Bibliotecología }\end{array}$ \\
\hline México & $\begin{array}{l}\text { Bibliotecología (2) } \\
\text { Filosofía y Letras (2) } \\
\text { Humanidades (1) } \\
\text { Ciencias de la Información (1) }\end{array}$ & $\begin{array}{l}\text { Filosofía y Letras } \\
\text { Bibliotecología }\end{array}$ \\
\hline Paraguay & $\begin{array}{l}\text { Politécnica } \\
\text { Bibliotecología (1) } \\
\text { Bibliotecología y Ciencias de la Información (1) } \\
\text { Humanidades (1) } \\
\text { Letras y Ciencias Humanas (2) }\end{array}$ & $\begin{array}{l}\text { Letras y Ciencias Humanas } \\
\text { Bibliotecología y Ciencias de la } \\
\text { Información }\end{array}$ \\
\hline Uruguay & $\begin{array}{l}\text { Información y Comunicación (1) } \\
\text { Información (1) } \\
\text { Bibliotecología y Ciencias Afines }\end{array}$ & $\begin{array}{l}\text { Politenica } \\
\text { Bibliotecología y Archivología (2) }\end{array}$ \\
\hline Venezuela & $\begin{array}{l}\text { Información } \\
\text { Biblecología }\end{array}$ \\
\hline
\end{tabular}

Tabla 3. Áreas del conocimiento de las que depende la formación bibliotecológica en cada uno de los países que conforman la muestra de la investigación Fuente: Múnera Torres, 2016: 148-160

Según la información de las Tablas 2 y 3, se observa que no obstante visualizar diferentes campos del saber de los cuales depende la formación bibliotecológica, existen países en los que la bibliotecología, la biblioteconomía, la ciencia de la información y la documentación se constituyen también en áreas de las cuales depende dicha formación, resultado que evidencia el papel de la bibliotecología como soporte de conocimiento frente a su misma formación profesional.

\section{DENOMINACIONES DE LA FORMACIÓN BIBLIOTECOLÓGICA en AmÉrica Latina}

La variación terminológica se manifiesta en el ámbito de las ciencias sociales y humanas de manera más recurrente y consiste en otorgar diferentes nombres a una misma realidad o a una misma denominación y puede corresponder a nociones diferentes, a nivel general o específico, de acuerdo con la definición que aporta Mónica Zurbano, citando a Jean-Claude Corbeil: "Es así que "diferentes denominaciones pueden corresponder más o menos a una 
misma noción (concurrencia terminológica) o una misma denominación puede corresponder a nociones diferentes, en general o en parte (polisemia terminológica) (Corbeil, 1988:57)" (2012: 3 ).

El fenómeno de la variación terminológica se presenta en la bibliotecología y desde hace algún tiempo viene manifestándose la inquietud sobre la variedad de nombres que se le da a la formación bibliotecológica, como lo evidencian diversos estudiosos. Por ejemplo, José López Yepes señala:

Creo que existe un auténtico conflicto conceptual y terminológico sobre ello como lo muestra el hecho de que el problema sigue siendo objeto de estudio en multitud de publicaciones seriadas científicas de nuestra especialidad y aquí arranca el primer conflicto: ¿cómo llamar a esta disciplina o tal vez, conjunto de disciplinas?: ¿Bibliotecología? ¿Biblioteconomía? ¿Documentación? ¿Ciencia de la Información? ¿Ciencia de los Bienes Culturales? ¿Y cómo llamar de modo unánime a los componentes, factores y aspectos diversos de las actividades documentales como, por ejemplo, al profesional? ¿Bibliotecario, bibliotecólogo, documentalista, profesional de la documentación, profesional de la información... ? Esto es algo que no ocurre en las disciplinas consagradas como Historia, Medicina, Química, Filosofía, Derecho, etc. (2009: 437-438)

Lo planteado anteriormente se manifiesta al apreciar la variedad de nombres que recibe la formación bibliotecológica tanto en diferentes universidades como en América Latina, y es que justo con la puesta en escena de nuevos soportes del saber y el desarrollo de la ciencia y la técnica se motiva el surgimiento de áreas como la documentación. Además, el desarrollo de la informática, las tecnologías de la información y la comunicación y una serie de innovaciones tecnológicas en la segunda mitad del siglo XX impulsan lo que se conoce como la sociedad de la información y del conocimiento, que junto con el Internet brindan paso a nuevas formas de producción del saber, con lo cual se originan nuevos soportes y denominaciones del desempeño del profesional de la bibliotecología. Por esta razón, surgen nuevas opciones de llamar a la formación bibliotecológica tales como la ciencia de la información, las ciencias de la información, y su articulación con áreas como la bibliotecología, la biblioteconomía y la documentación.

Con relación a lo anterior, Agustín Gutiérrez Chiñas y Miguel Ángel Rendón expresan lo siguiente:

Ante los cambios y la evolución que provocan la ciencia, la tecnología y la moda, de acuerdo con Miguel Ángel Rendón, es necesario e "importante conservar la identidad disciplinar para no diluirse en esas transformaciones y caer en la 
esquizofrenia de poseer diferentes nombres y personalidades. En el desarrollo de la persona humana, por ejemplo, encontramos cambios, en ocasiones muy significativos, basta con ver una fotografía de cuando teníamos tres meses de edad y una contemporánea, pero conservamos la mismidad, la identidad propia, $[\ldots]$ de cada uno. Imaginemos qué pasaría si una persona se presenta una vez como Juan, luego como Pedro, después se llama Andrés, luego Santiago y en otra ocasión se llama Rosita. Sencillamente aquellos con quienes se relaciona esta persona no sabrían realmente quien es ella e incluso la tacharían de poco seria.”(Rendón Rojas, 2008:165). Algo similar sucede con una ciencia cuando se dispersa en los nombres con los que se identifica, con lo cual, de esta manera, pierde su identidad, coherencia y articulación de los elementos que la integran, lo que trae consigo, de manera automática, la pérdida o disminución de su seriedad y formalidad ante la comunidad científica en general. (Gutiérrez Chiñas, 2014: 43-44)

El fenómeno de la variación terminológica de la formación bibliotecológica contribuye también con la pérdida de la identidad de este ámbito del conocimiento, al ser abordado desde diferentes perspectivas y nombres. Esto, por supuesto, genera dispersión y problemas de homologación de contenidos al momento de realizar procesos de formación interuniversitaria e interinstitucional. Al respecto Jaime Ríos Ortega señala:

Una de las preocupaciones actuales para la formación de los bibliotecólogos se relaciona con la propuesta de establecer un cuerpo unificado de teorías que identifiquen de modo único al bibliotecólogo, con lo que se busca evitar la desintegración de la identidad profesional apoyándose en diversas escuelas de pensamiento: Documentación, Ciencias de la Información, Bibliotecología, etcétera. (2008: 20)

Como se puede evidenciar, la variación terminológica en el ámbito de la formación bibliotecológica es una realidad que se manifiesta en la actualidad y se logra identificar al observar los nombres que recibe dicha formación en diferentes universidades de América Latina.

Con esta investigación se logra identificar que aun cuando han surgido diversas maneras de nombrar a la formación bibliotecológica en América Latina, se aprecia una mayoría de países y universidades en las que se utiliza la denominación de biblioteconomía y bibliotecología para designar dicha formación. Se observa además cómo en algunos casos aparecen las denominaciones de bibliotecología y biblioteconomía de forma independiente, mientras que en otros aparecen acompañadas con nombres como la archivología, la ciencia de la información, las ciencias de la información, la documentación, estudios de la información, entre otros. 
La variable anterior consiste en identificar cómo el área del conocimiento de la que más depende la formación bibliotecológica en América Latina, de acuerdo con la muestra definida para la investigación, es la ciencia de la información. En contraste, al explorar por las denominaciones que más recibe la formación bibliotecológica en América Latina, se encuentra que un solo programa de formación bibliotecológica de la misma muestra recibe el nombre de ciencia de la información-bibliotecología.

El panorama de la denominación de la formación bibliotecológica en América Latina se ilustra en la Tabla 4.

\begin{tabular}{|c|c|}
\hline \multicolumn{2}{|c|}{ Denominación de la formación bibliotecológica en América Latina } \\
\hline Nombres de los programas & Cantidad \\
\hline Bibliotecología & 6 \\
\hline Bibliotecología y Archivología & 1 \\
\hline Bibliotecología y Ciencia de la Información & 2 \\
\hline Bibliotecología y Ciencias de la Información & 4 \\
\hline Bibliotecología y Documentación & 5 \\
\hline Bibliotecología y Estudios de la Información & 1 \\
\hline Biblioteconomía & 17 \\
\hline Biblioteconomía y Ciencia de la Información & 1 \\
\hline Biblioteconomía, Ciencias de la Información y de la Documentación & 1 \\
\hline Biblioteconomía y Documentación & 3 \\
\hline Ciencia de la Información-Bibliotecología & 1 \\
\hline Ciencias de la Información & 3 \\
\hline Ciencias de la Información Documental & 1 \\
\hline Gestión de la Información & 3 \\
\hline Sistemas de Información y Documentación & 1 \\
\hline
\end{tabular}

Tabla 4. Denominación de la formación bibliotecológica en América Latina Fuente: Múnera Torres, 2016: 162

Por otra parte, y para contrastar el resultado general en cuanto a la denominación de la formación bibliotecológica en América Latina, se procedió a indagar en cada uno de los países y universidades que conforman la muestra la denominación que mayor uso presenta en cada país, de acuerdo con la siguiente información: 
- Uso de los términos bibliotecología o biblioteconomía como denominación específica de la formación bibliotecológica.

- Uso de los términos bibliotecología o biblioteconomía, acompañados por denominaciones como archivología, ciencia de la información, ciencias de la información, documentación, estudios de la información, entre otras, como nombres de la formación bibliotecológica.

- Uso de los términos ciencia de la información, ciencias de la información, ciencias de la información documental, gestión de información, sistemas de información, gestión de información, como nombres de la formación bibliotecológica.

Luego de identificar la denominación que mayor uso presenta en la formación bibliotecológica de América Latina, se llega a la conclusión de que los nombres bibliotecología y biblioteconomía son los que más se manifiestan dentro de la muestra de universidades objeto de estudio en la investigación, tal como se ilustra en la Tabla 5.

\begin{tabular}{|c|c|c|}
\hline País & Denominaciones de la formación bibliotecológica & Denominación más usada \\
\hline Argentina & $\begin{array}{l}\text { Bibliotecología y Ciencia de la Información (2) } \\
\text { Bibliotecología y Ciencias de la Información (2) } \\
\text { Bibliotecología y Documentación (1) }\end{array}$ & Bibliotecología \\
\hline Brasil & $\begin{array}{l}\text { Biblioteconomía (17) } \\
\text { Biblioteconomía y Ciencia de la Información (1) } \\
\text { Biblioteconomía y Documentación (2) } \\
\text { Ciencias de la Información y la Documentación (2) } \\
\text { Gestión de Información (3) }\end{array}$ & Biblioteconomía \\
\hline Chile & Bibliotecología (1) & Bibliotecología \\
\hline Colombia & $\begin{array}{l}\text { Bibliotecología (1) } \\
\text { Ciencia de la Información-Bibliotecología (1) } \\
\text { Sistemas de Información y Documentación (1) }\end{array}$ & Bibliotecología \\
\hline Costa Rica & Bibliotecología y Documentación (2) & Bibliotecología y Documentación \\
\hline Guatemala & Bibliotecología (1) & Bibliotecología \\
\hline México & $\begin{array}{l}\text { Bibliotecología (1) } \\
\text { Bibliotecología y Ciencia de la Información (1) } \\
\text { Bibliotecología y Estudios de la Información (1) } \\
\text { Ciencias de la Información Documental (1) }\end{array}$ & Bibliotecología \\
\hline Paraguay & Ciencias de la Información (1) & Ciencias de la Información \\
\hline Perú & $\begin{array}{l}\text { Ciencias de la Información (1) } \\
\text { Bibliotecología y Ciencias de la Información (1) }\end{array}$ & Ciencias de la Información \\
\hline
\end{tabular}




\begin{tabular}{|l|l|l|}
\hline Uruguay & Bibliotecología (1) & Bibliotecología \\
\hline Venezuela & Bibliotecología y Archivología (2) & Bibliotecología y Archivología \\
\hline
\end{tabular}

Tabla 5. Relación de denominaciones de la formación bibliotecológica en los países de América Latina que forman parte de la muestra de la investigación

Fuente: elaboración propia

\section{CONSIDERACiones Finales}

Sin lugar a dudas, la formación bibliotecológica manifiesta una serie de cambios en su proceso de evolución, acordes con el surgimiento de nuevos paradigmas, tendencias e influencias de orden tecnológico, cognoscitivo, económico, social, académico, educativo, político, que de una u otra forma han perfilado el desempeño profesional bibliotecológico a la luz de fenómenos como el de la sociedad de la información y del conocimiento, la globalización, la explosión de la información, la constitución de la biblioteca como impulsadora de procesos de desarrollo social, educativo y político dentro de las comunidades, el desarrollo del emprendimiento en el campo bibliotecológico, así como la investigación bibliotecológica y demás componentes que conforman las nuevas dimensiones de la educación bibliotecológica actual.

Cuando se abordan las áreas del conocimiento de las cuales depende la formación bibliotecológica en América Latina, acorde con la muestra de universidades que se conformó, se identifica que las áreas del conocimiento de las cuales depende más dicha formación son la ciencia de la información, la bibliotecología, la biblioteconomía, la filosofía y letras y las humanidades. En este resultado es importante tener en cuenta que más de un cincuenta por ciento de la muestra está conformado por universidades del Brasil, situación que influye mucho en el resultado del estudio de esta variable.

$\mathrm{Al}$ contrastar el anterior resultado con las áreas del conocimiento de las que depende la formación bibliotecológica en cada uno de los países que conforman la muestra, se logra identificar a la bibliotecología, la biblioteconomía y campos afines como áreas de la cuales depende dicha formación. Esta situación evidencia el peso académico de la bibliotecología como área del conocimiento de la cual depende su misma formación profesional.

La variación en la denominación de la formación bibliotecológica en América Latina también se constituye en uno de los aspectos que mayor inquietud genera en el ámbito de la investigación bibliotecológica, debido a la diversidad de términos con los que se identifica el ejercicio profesional de la bibliotecología dentro del contexto de la sociedad actual. Sin embargo, y 
luego de realizar un estudio comparativo de los nombres que se le otorgan a la formación bibliotecológica en las universidades objeto de estudio, se logra identificar una mayoría en las que se utiliza la denominación de biblioteconomía y bibliotecología para designar dicha formación. Se observa además cómo en algunos casos aparecen las denominaciones de bibliotecología y biblioteconomía de forma independiente; sin embargo, en otros aparecen acompañadas las denominaciones bibliotecología o biblioteconomía con nombres como la archivología, la ciencia de la información, las ciencias de la información, la documentación, estudios de la información, entre otros.

No obstante existir diferentes propuestas de denominación de la formación bibliotecológica en América Latina, como resultado de la introducción de nuevos paradigmas que se manifiestan desde el siglo XX los términos de bibliotecología y biblioteconomía aún se incluyen en los nombres de las ofertas de formación en este ámbito del saber, lo cual indica que la misma bibliotecología o biblioteconomía, como se asume en algunos países de América Latina y Europa, se continúa utilizando como una denominación muy demandada para este tipo de formación y desempeño profesional.

\section{REFERENCIAS}

Escalona Ríos, Lina. 2011. La evaluación de la educación bibliotecológica en América Latina. México: UNAM, CUIB.

Gutiérrez Chiñas, Agustín. 2014. "Principales denominaciones que se utilizan para nombrar los conceptos de la bibliotecología / documentación / ciencia de la información”, en El problema del lenguaje en la bibliotecología / ciencia de la información / documentación: un acercamiento filosófico-teórico, M. Á. Rendón Rojas (coord.), 33-47. México: UNAM, IIBI.

López Yepes, José. 2009. "Algunos problemas terminológicos en el dominio de la bibliotecología y documentación. Una Babel terminológica-conceptual”, en I Simposio Internacional sobre organización del conocimiento: bibliotecología y terminología, 27 al 29 de agosto de 2007, C. Naumis Peña (coord.), 447-477. México: UNAM, Centro Universitario de Investigaciones Bibliotecológicas, CUIB.

López Yepes, José. 2004. Diccionario Enciclopédico de Ciencias de la Documentación. Madrid: Síntesis.

Múnera Torres, María Teresa. 2014. "Terminología de la bibliotecología en América Latina: un avance de investigación”, en Naturaleza y método de la investigación bibliotecológica y de la información, J. Ríos Ortega y C. A. Ramírez Velásquez (coords.), 111-129. México: UNAM, IIBI.

Múnera Torres, María Teresa. 2016. "La terminología de la bibliotecología en América Latina: un estudio comparativo”. Tesis, Doctorado en Información y Documentación, Universidad de Zaragoza.

Orera Orera, Luisa. 1995. "Evolución histórica del concepto de biblioteconomía". Revista General de Información y Documentación (Madrid) 5 (2): 73-89. 
Orera Orera, Luisa. 2002. Manual de Biblioteconomía. Madrid: Síntesis.

Ríos Ortega, Jaime. 2008. Didáctica de la Bibliotecología: Teoría y principios desde la enseñanza de la ciencia. México: UNAM, CUIB.

Setién Quesada, Emilio. 2002. "Posibles derroteros de la teoría bibliotecológica en el siglo XXI”. SCIRE (Zaragoza) 2: 33-42.

Universidad de Buenos Aires. Facultad de Filosofía y Letras. Departamento de Bibliotecología y Ciencia de la Información. 2010. Presentación de la carrera. Fecha de consulta: 15 de mayo de 2014. bibliotecología.filo.uba.ar

Zurbano López, Mónica. 2012. Reflexiones en torno a la variación terminológica y su importancia para el traductor jurídico, presentado en Octavo Simposio de Traducción, Terminología e Interpretación, Habana, ACTI y CTIC, 11, 12 y 13 de diciembre. Fecha de consulta: 14 de enero de 2015. http://www.cttic.org/ACTI/2012/Actes/MZurbano.pdf

Para citar este texto:

Múnera Torres, María Teresa. 2018. "Algunas tendencias de la formación bibliotecológica en América Latina”. Investigación Bibliotecológica: archivonomía, bibliotecología e información 32 (74): 101-122. http://dx.doi.org/10.22 201/iibi.24488321xe.2018.74.57912 


\section{Anexo}

\begin{tabular}{|c|c|c|}
\hline \multicolumn{3}{|c|}{$\begin{array}{l}\text { Relaciones de países, universidades y programas de formación bibliotecológica } \\
\text { en América Latina }\end{array}$} \\
\hline País & Uninversidad y unidad académica & Programas \\
\hline \multirow[t]{3}{*}{ 1. Argentina } & $\begin{array}{l}\text { 1. Universidad de Buenos Aires } \\
\text { Facultad de Filosofía y Letras } \\
\text { Departamento de Bibliotecología y } \\
\text { Ciencia de la Información }\end{array}$ & $\begin{array}{l}\text { 1. Licenciatura en Bibliotecología y } \\
\text { Ciencia de la Información } \\
\text { 2. Profesorado en Bibliotecología y } \\
\text { Ciencia de la Información }\end{array}$ \\
\hline & $\begin{array}{l}\text { 2. Universidad Nacional de Córdoba } \\
\text { Facultad de Filosofía y Letras } \\
\text { Escuela de Bibliotecología }\end{array}$ & $\begin{array}{l}\text { 3. Licenciatura en Bibliotecología y } \\
\text { Documentación }\end{array}$ \\
\hline & $\begin{array}{l}\text { 3. Universidad Nacional de La Plata } \\
\text { Facultad de Humanidades y Ciencias de } \\
\text { la Educación } \\
\text { Departamento de Bibliotecología }\end{array}$ & $\begin{array}{l}\text { 4. Licenciatura en Bibliotecología y } \\
\text { Ciencias de la Información } \\
\text { 5. Profesorado en Bibliotecología y } \\
\text { Ciencias de la Información }\end{array}$ \\
\hline \multirow[t]{6}{*}{ 2. Brasil } & $\begin{array}{l}\text { 1. Pontificia Universidad Católica de } \\
\text { Campinas } \\
\text { Centro de Ciencias Humanas y Sociales } \\
\text { Aplicadas } \\
\text { Facultad de Biblioteconomía }\end{array}$ & 1. Biblioteconomía \\
\hline & $\begin{array}{l}\text { 2. Universidad de Brasilia } \\
\text { Facultad de Ciencia de la Información }\end{array}$ & 2. Biblioteconomía \\
\hline & $\begin{array}{l}\text { 3. Universidad del Estado de Santa } \\
\text { Catalina } \\
\text { Centro de Ciencias Humanas y de } \\
\text { Educación } \\
\text { Departamento de Biblioteconomía }\end{array}$ & 3. Biblioteconomía \\
\hline & $\begin{array}{l}\text { 4. Universidad Estadual de Londrina } \\
\text { Centro de Educación, Comunicación } \\
\text { y Artes } \\
\text { Depto. de Ciencias de la Información }\end{array}$ & 4. Biblioteconomía \\
\hline & $\begin{array}{l}\text { 5. Universidad Estadual Paulista } \\
\text { Facultad de Filosofía y Ciencias } \\
\text { Departamento de Ciencias de la } \\
\text { Información }\end{array}$ & 5. Biblioteconomía \\
\hline & $\begin{array}{l}\text { 6. Universidad Federal de Alagoas } \\
\text { Instituto de Ciencias Humanas, } \\
\text { Comunicación y Arte }\end{array}$ & 6. Biblioteconomía \\
\hline
\end{tabular}




\begin{tabular}{|c|c|}
\hline $\begin{array}{l}\text { 7. Universidad Federal de Bahía } \\
\text { Instituto de Ciencias de la Información } \\
\text { Departamento de Información } \\
\text { y Documentación }\end{array}$ & 7. Biblioteconomía y Documentación \\
\hline $\begin{array}{l}\text { 8. Universidad Federal de Ceará } \\
\text { Centro de Humanidades } \\
\text { Departamento de Ciencias } \\
\text { de la Información }\end{array}$ & 8. Biblioteconomía \\
\hline $\begin{array}{l}\text { 9. Universidad Federal del Estado de Río } \\
\text { de Janeiro } \\
\text { Centro de Ciencias Humanas y Sociales } \\
\text { Escuela de Biblioteconomía } \\
\text { 10. Universidad Federal Fluminense } \\
\text { Instituto de Arte y Comunicación Social } \\
\text { Biblioteconomía y Documentación }\end{array}$ & $\begin{array}{l}\text { 9. Bachillerato en Biblioteconomía } \\
\text { 10. Licenciatura en Biblioteconomía } \\
\text { 11. Biblioteconomía y } \\
\text { Documentación }\end{array}$ \\
\hline $\begin{array}{l}\text { 11. Universidad Federal de Goias } \\
\text { Facultad de Comunicación } \\
\text { y Biblioteconomía } \\
\text { 12. Universidad Federal de Matto } \\
\text { Grosso } \\
\text { Instituto de Ciencias Humanas } \\
\text { y Sociales }\end{array}$ & $\begin{array}{l}\text { 12. Biblioteconomía } \\
\text { 13. Gestión de Información } \\
\text { 14. Biblioteconomía }\end{array}$ \\
\hline $\begin{array}{l}\text { 13. Universidad Federal de Para Ufpa } \\
\text { Instituto de Ciencias Sociales, Aplicadas } \\
\text { Facultad de Biblioteconomía }\end{array}$ & 15. Biblioteconomía \\
\hline $\begin{array}{l}\text { 14. Universidad Federal de Paraná } \\
\text { Sector Ciencias Sociales Aplicadas } \\
\text { Departamento de Ciencia y Gestión } \\
\text { de la Información }\end{array}$ & 16. Gestión de Información \\
\hline $\begin{array}{l}\text { 15. Universidad Federal de Pernambuco } \\
\text { Centro de Artes y Comunicación } \\
\text { Departamento de Ciencia de } \\
\text { Información } \\
\text { 16. Universidad Federal de Rio Grande } \\
\text { Instituto de Ciencias Humanas y de la } \\
\text { Información }\end{array}$ & $\begin{array}{l}\text { 17. Biblioteconomía } \\
\text { 18. Gestión de Información } \\
\text { 19. Biblioteconomía }\end{array}$ \\
\hline $\begin{array}{l}\text { 17. Universidad Federal de Rio Grande } \\
\text { del Norte } \\
\text { Centro de Ciencias Sociales y Aplicadas } \\
\text { Departamento de Biblioteconomía }\end{array}$ & 20. Biblioteconomía \\
\hline
\end{tabular}




\begin{tabular}{|c|c|c|}
\hline & $\begin{array}{l}\text { 18. Universidad Federal de Rio } \\
\text { Grande del Sur } \\
\text { Facultad de Biblioteconomía } \\
\text { y Comunicación } \\
\text { Departamento de Ciencias de la } \\
\text { Información }\end{array}$ & 21. Biblioteconomía \\
\hline & $\begin{array}{l}\text { 19. Universidad Federal de San Carlos } \\
\text { Centro de Educación y Ciencias } \\
\text { Humanas } \\
\text { Departamento de Ciencia de la } \\
\text { Información }\end{array}$ & $\begin{array}{l}\text { 22. Biblioteconomía y Ciencia de la } \\
\text { Información }\end{array}$ \\
\hline & $\begin{array}{l}\text { 20. Universidad Federal de Santa } \\
\text { Catalina } \\
\text { Centro de Ciencias de la Educación } \\
\text { Departamento de Ciencia de la } \\
\text { Información }\end{array}$ & 23. Biblioteconomía \\
\hline & $\begin{array}{l}\text { 21. Universidad de São Paulo } \\
\text { Escuela de Comunicaciones y Artes } \\
\text { Departamento de Biblioteconomía y } \\
\text { Documentación }\end{array}$ & $\begin{array}{l}\text { 24. Bachillerato en Biblioteconomía } \\
\text { y Documentación }\end{array}$ \\
\hline & $\begin{array}{l}\text { 22. Universidad de São Paulo } \\
\text { Facultad de Filosofía, Ciencias y Letras } \\
\text { de Ribeirão Preto } \\
\text { Departamento de Educación, Informa- } \\
\text { ción y Comunicación }\end{array}$ & $\begin{array}{l}\text { 25. Bachillerato en Biblioteconomía, } \\
\text { Ciencias de la Información y la } \\
\text { Documentación }\end{array}$ \\
\hline 3. Chile & $\begin{array}{l}\text { 1. Universidad de Playa Ancha } \\
\text { Facultad de Ciencias Sociales } \\
\text { Departamento de Ciencias de la } \\
\text { Documentación }\end{array}$ & 1. Bibliotecología \\
\hline \multirow[t]{3}{*}{ 4. Colombia } & $\begin{array}{l}\text { 1. Pontificia Universidad Javeriana } \\
\text { Facultad de Comunicación y Lenguaje } \\
\text { Departamento de Ciencia de la } \\
\text { Información }\end{array}$ & $\begin{array}{l}\text { 1. Ciencia de la Información- } \\
\text { Bibliotecología }\end{array}$ \\
\hline & $\begin{array}{l}\text { 2. Universidad de Antioquia } \\
\text { Escuela Interamericana de } \\
\text { Bibliotecología }\end{array}$ & 2. Bibliotecología \\
\hline & $\begin{array}{l}\text { 3. Universidad de La Salle } \\
\text { Facultad de Ciencias Económicas } \\
\text { y Sociales }\end{array}$ & 3. Sistemas de Información \\
\hline 5. Costa Rica & $\begin{array}{l}\text { 1. Universidad Nacional de Costa Rica } \\
\text { Facultad de Filosofía y Letras } \\
\text { Escuela de Bibliotecología, } \\
\text { Documentación e Información }\end{array}$ & $\begin{array}{l}\text { 1. Bachillerato y Licenciatura en Bi- } \\
\text { bliotecología y Documentación con } \\
\text { énfasis en Gestión de la Información } \\
\text { con Salida Lateral de Diplomado } \\
\text { 2. Bachillerato y Licenciatura en } \\
\text { Bibliotecología y Documentación } \\
\text { con Énfasis en Tecnología de la } \\
\text { Información y de la Comunicación }\end{array}$ \\
\hline
\end{tabular}




\begin{tabular}{|c|c|c|}
\hline 6. Guatemala & $\begin{array}{l}\text { 1. Universidad de San Carlos } \\
\text { Facultad de Humanidades } \\
\text { Escuela de Bibliotecología }\end{array}$ & 1. Licenciatura en Bibliotecología \\
\hline \multirow[t]{4}{*}{ 7. México } & $\begin{array}{l}\text { 1. Universidad Autónoma de San } \\
\text { Luis Potosí } \\
\text { Escuela de Ciencias de la Información }\end{array}$ & 1. Licenciatura en Bibliotecología \\
\hline & $\begin{array}{l}\text { 2. Universidad del Estado de México } \\
\text { Facultad de Humanidades }\end{array}$ & $\begin{array}{l}\text { 2. Licenciatura en Ciencias de la } \\
\text { Información Documental }\end{array}$ \\
\hline & $\begin{array}{l}\text { 3. Universidad Nacional Autónoma } \\
\text { de México } \\
\text { Facultad de Filosofía y Letras } \\
\text { Colegio de Bibliotecología }\end{array}$ & $\begin{array}{l}\text { 3. Licenciatura en Bibliotecología y } \\
\text { Estudios de la Información }\end{array}$ \\
\hline & $\begin{array}{l}\text { 4. Universidad de Nuevo León } \\
\text { Facultad de Filosofía y Letras } \\
\text { Colegio de Bibliotecología }\end{array}$ & $\begin{array}{l}\text { 4. Licenciatura en Bibliotecología y } \\
\text { Ciencias de la Información }\end{array}$ \\
\hline 8. Paraguay & $\begin{array}{l}\text { 1. Universidad Nacional de Asunción } \\
\text { Facultad Politécnica }\end{array}$ & $\begin{array}{l}\text { 1. Licenciatura en Ciencias de la } \\
\text { Información }\end{array}$ \\
\hline \multirow[t]{2}{*}{ 9. Perú } & $\begin{array}{l}\text { 1. Pontificia Universidad Católica } \\
\text { del Perú } \\
\text { Facultad de Letras y Ciencias Humanas } \\
\text { Departamento de Humanidades } \\
\text { Sección de Bibliotecología y Ciencias de } \\
\text { la Información }\end{array}$ & $\begin{array}{l}\text { 1. Licenciatura en Ciencias de la } \\
\text { Información }\end{array}$ \\
\hline & $\begin{array}{l}\text { 2. Universidad Nacional Mayor de San } \\
\text { Marcos } \\
\text { Facultad de Letras y Ciencias Humanas } \\
\text { Escuela de Bibliotecología }\end{array}$ & $\begin{array}{l}\text { 2. Bibliotecología y Ciencias de la } \\
\text { Información }\end{array}$ \\
\hline 10. Uruguay & $\begin{array}{l}\text { 1. Universidad de la República } \\
\text { Facultad de Información y Comunicación } \\
\text { Instituto de Información } \\
\text { Escuela Universitaria de Bibliotecología } \\
\text { y Ciencias Afines }\end{array}$ & 1. Licenciatura en Bibliotecología \\
\hline \multirow[t]{2}{*}{ 11. Venezuela } & $\begin{array}{l}\text { 1. Universidad Central de Venezuela } \\
\text { Facultad de Humanidades y Educación } \\
\text { Escuela de Bibliotecología y Archivología }\end{array}$ & $\begin{array}{l}\text { 1. Licenciatura en Bibliotecología y } \\
\text { Archivología }\end{array}$ \\
\hline & $\begin{array}{l}\text { 2. Universidad de Zulia } \\
\text { Facultad de Humanidades y Educación } \\
\text { Escuela de Bibliotecología y Archivología }\end{array}$ & $\begin{array}{l}\text { 2. Licenciatura en Bibliotecología y } \\
\text { Archivología }\end{array}$ \\
\hline
\end{tabular}

Relación de universidades y programas de formación bibliotecológica que conforman la muestra de la investigación titulada "La terminología de la bibliotecología en América Latina: un estudio comparativo" 